\title{
Structure Factor of Charged Microemulsion Nanodroplets Decorated with Telechelic Polymers: Experimental and Numerical Study
}

\author{
Rachid Ahfir, Mohammed Filali* \\ Laboratory of Theoretical and Applied Physics (LPTA), Department of Physics, Faculty of Sciences Dhar El Mahraz, \\ Sidi Mohamed Ben Abdellah University, Fez, Atlas. BP 1796, Morocco
}

*Corresponding author: E-mail: mohammed.filali@usmba.ac.ma; Tel.: (212) 665845614

DOI: $10.5185 /$ amlett.2020.081548

We investigate thermodynamic and structural properties of positively charged $\mathrm{O} / \mathrm{W}$ microemulsion spherical nanodroplets, suspended in salt water and decorated with telechelic polymers PEO-m and PEO-2m at low and high volume fraction $\Phi(6.98 \%, 26.5 \%)$ by using Small Angle Neutron Scattering (SANS). We describe and propose an effective pair potential interaction between charged colloidal particles in solution both with and without added telechelic polymers PEO-m or PEO-2m.We solve Ornstein-Zernicke (OZ) integral equation in the Hypernetted Chain $(\mathrm{HNC})$ closure relation to obtain the pair correlation function $g(r)$ and the structure factor $\mathrm{S}(\mathrm{q})$. A good agreement is found between the experimental and numerical spectra assuming the interaction potentials to be the sum of attractive and repulsive contributions in terms of decoration or bridging of the nanodroplets by the PEO polymer chains.

\section{Introduction}

The interactions between surfactant molecules and synthetic polymers in aqueous solutions are of importance to many practical applications, e.g., in the food, pharmaceutical, biotechnology, nanotechnology, medicine, pharmacology, cosmetics, agriculture, water purification, electronic, optoelectronic, and enhanced oil recovery and consumer products industries [1-3].In these mixed systems (surfactant and polymers), the nature of the polymers plays a very important role in the interaction between them and the surfactant film [4]. Asakura and Oosawa were the first to investigate the attractive depletion-interaction between two particles immersed in a dilute solution of non-adsorbing polymer chains $[\mathbf{5 , 6}]$. Alternately, repulsion between colloidal particles with grafted or adsorbed polymers on their surfaces can be attributed to osmotic interactions between segments of the polymers. Upon approach of two covered particles, the polymers that constitute the layers start to repeal each other by entropic restriction related to the confinement of available spatial configurations [7-9]. These phenomena are often examined using either scaling analysis [10] or via computer simulations $[\mathbf{1 1}, \mathbf{1 2}]$.

The interactions between nanodroplets of oil-in-water $(\mathrm{O} / \mathrm{W})$ microemulsion decorated and/or linked by telechelic polymers have received considerable attention in recent years [13]. The main advantage of these systems over others, like binary solutions of associative polymers, is that the number density of nodes (nanodroplets) and the number of polymers per nanodroplet may be controlled independently. The first theoretical approach was proposed by Milner and Witten, who deployed selfconsistent field theories to telechelic polymer brushes between flat plates and found a weak attraction (less than $0.1 \mathrm{k}_{\mathrm{B}} \mathrm{T}$ per chain) due to the bridging chains [14]. Using an approach derived from Milner and Witten, Meng and Russel [15] found an attractive interaction for telechelic chains between two flat plates analytically. This behavior has been supported by experimental results $[16, \mathbf{1 7}]$. Other effort has been put into the description of the adsorption of telechelic polymers between two solid parallel flat plates by using Monte Carlo [18] and molecular dynamics simulations [19]. With regard to the curved surfaces, to our knowledge, the best description of the potentials between two spheres linked by telechelic polymers is given by Bhatia and Russel [20]. These authors used the diffusion equation and obtained numerical results. Porte et. al., [21] employed Bahtia and Russel's model to estimate the bridging interactions between droplets due to telechelic linkers and to compare to the experimental results. They found a slight disagreement between the calculated and experimental spectra at low q scattering. Similar disagreement has also been observed for a system composed of nanoparticles dispersed in high polymer concentration solution [22]. Despite a fairly good semi quantitative agreement between the theory and the experiments, the picture is yet far from being complete $[\mathbf{2 3}, \mathbf{2 4}]$. Then, to obtain a precise and qualitative picture of the microemulsion nanodroplet organization with the addition of a hydrophobically modified polymer at one or both ends, we propose a model on the shape of the interaction potential that describes these systems. 


\section{Advanced Materials Letters www.vbripress.com/aml}

In this study, we planned to describe and model the interactions between positively charged $\mathrm{O} / \mathrm{W}$ microemulsion spherical nanodroplets in the presence of the telechelic polymers. For this, we use a microemulsion of decane nanodroplets stabilized in brine water by a monolayer of the ionic surfactant $\mathrm{CpCl}$ and cosurfactant octanol. To this microemulsion, we add a telechelic polymer PEO-m or PEO- $2 \mathrm{~m}$. We will note PEO-m or PEO-2m, the PEO, water-soluble chain poly (ethylene oxide), carrying one or two hydrophobic ends $\left(\mathrm{C}_{12} \mathrm{H}_{25}\right)$. The PEO-m simply decorates the nanodroplets, while the PEO-2m has two possible configurations: bridging two different nanodroplets or decorating them. The paper is organized as follows: In section 2, materials and preparation of the samples (bare microemulsion, with PEO-m or PEO- $2 \mathrm{~m}$ ) are presented. The structure of the mixture system has been studied by SANS. In section 3, the theoretical background is proposed, through the pair potential and the theory of Integral Equations Method. In section 4, we first check that the size and shape of the nanodroplets remain constant upon addition of moderate amounts of polymers by the invariance of the form factor. Then, we study the effect of an addition of modified polymers on the interactions between nanodroplets by analyzing $\mathrm{g}(\mathrm{r})$ and $\mathrm{S}(\mathrm{q})$. We show the strengthening of the repulsive interaction between nanodroplets by adding PEO-m on the bare microemulsion, while the addition of PEO-2m introduces an effective attractive interaction due to the bridging. We have modeled these interactions by appropriate potentials and found a good agreement between the experimental and numerical spectra for all cases studied.We conclude our results with the thermodynamic quantity (the excessive internal energy) discussed in terms of the volume fraction and the polymer concentration.

\section{Experimental section}

\section{Preparation of the microemulsion}

Cetylpyridinium Chloride $\quad\left[\mathrm{H}_{3} \mathrm{C}-\left(\mathrm{CH}_{2}\right)_{15}\right] \mathrm{C}_{5} \mathrm{H}_{5} \mathrm{~N}^{+} \mathrm{Cl}^{-}$ $(\mathrm{CpCl})$, Decane $\left[\mathrm{H}_{3} \mathrm{C}-\left(\mathrm{CH}_{2}\right)_{8} \mathrm{CH}_{3}\right]$ and Octanol $\left[\mathrm{H}_{3} \mathrm{C}-\right.$ $\left.\left(\mathrm{CH}_{2}\right)_{7}\right]-\mathrm{OH}$ from Fluca. Cetylpyridinium Chloride is purified by successive recrystallization in water and in acetone, octanol and decane are used as received. The poly (ethylene oxide) have been hydrophobically modified and purified in the laboratory using the method described in Refs. [25] and [26]. The molecular weight of the starting products is determined by size-exclusion chromatography. The hydrophobically modified poly (ethylene oxide) contains an isocyanate group between the alkyl chain $\left(\mathrm{C}_{12} \mathrm{H}_{25}\right)$ and the ethylene oxide chain. We assume that this isocyanate group belongs to the hydrophilic part of the copolymer. Poly (ethylene oxide) PEO-m is modified at one extremity only. PEO- $2 \mathrm{~m}$ is modified at both extremities. PEO-m is exactly half the PEO-2m (cf. Table 1). After modification, NMR using the method described in Ref. [27] determined the degree of substitution of hydroxyl groups. The degree of substitution is found to be greater than $98 \%$.

The microemulsion is a thermodynamically stable dispersion in water of oil nanodroplets surrounded by a surfactant film [28] O/W microemulsion. The spontaneous radius of curvature of the surfactant film, composed of a cationic surfactant $(\mathrm{CpCl})$ and a cosurfactant (Octanol), is adjusted by varying its composition. The ratio in weight of Octanol to $\mathrm{CpCl}$ is set equal to 0.25 . The ratio in weight of Decane to surfactant film is 0.62 . This ratio is chosen to be close to the emulsification failure limit, but slightly below this threshold. The line of the emulsification failure is the limit above which the microemulsion nanodroplets are saturated with oil and coexist with excess oil. On this line, the microemulsion nanodroplets have a radius corresponding to the spontaneous curvature radius of the surfactant film [29]. Under such conditions the microemulsion droplets are spheres of well-defined radius $[30,31]$, which can be diluted over a large concentration range [32]. In our case, the microemulsion could be diluted from $30 \%$ to $1 \%$ volume fraction.

Table 1. Molar mass and density of the components of the samples.

\begin{tabular}{lcc}
\hline Components & $\begin{array}{c}\text { Molar } \\
\text { mass } \\
\text { (Dalton) }\end{array}$ & $\begin{array}{c}\text { Density } \\
\left(\mathbf{g} / \mathbf{c m}^{\mathbf{3}}\right)\end{array}$ \\
\hline $\mathrm{H}_{2} \mathrm{O}$ & 18 & 1 \\
$\mathrm{D}_{2} \mathrm{O}$ & 20 & 1.105 \\
{$\left[\mathrm{H}_{3} \mathrm{C}\left(\mathrm{CH}_{2}\right)_{15}\right] \mathrm{C}_{5} \mathrm{H}_{5} \mathrm{~N}^{+} \mathrm{Cl}^{-}\left(\mathrm{C}_{\mathrm{p}} \mathrm{C} 1\right)$} & 339.5 & 1.656 \\
{$\left[\mathrm{H}_{3} \mathrm{C}\left(\mathrm{CH}_{2}\right)_{7}\right] \mathrm{OH}(\mathrm{Octanol})$} & 130 & 1.8 \\
{$\left[\mathrm{H}_{3} \mathrm{C}\left(\mathrm{CH}_{2}\right)_{8} \mathrm{CH}_{3}\right]($ Decane $)$} & 142 & 0.73 \\
$\left.\mathrm{H}_{3} \mathrm{C}\left(\mathrm{OCH}_{2} \mathrm{CH}_{2}\right)_{113} \mathrm{O}(\mathrm{CO}) \mathrm{NH}_{(}\left(\mathrm{CH}_{2}\right)_{11} \mathrm{CH}_{3}\right](\mathrm{PEO}-\mathrm{m})$ & 5200 & 1.2 \\
{$\left[\mathrm{CH}_{3}\left(\mathrm{CH}_{2}\right)_{11} \mathrm{NHCO}_{2} \mathrm{OCH} \mathrm{CH}_{2}\right)_{22} \mathrm{O}(\mathrm{CO}) \mathrm{NH}$} & 10400 & 1.2 \\
{$\left[\left(\mathrm{CH}_{2}\right)_{11} \mathrm{CH}_{3}\right](\mathrm{PEO}-2 \mathrm{~m})$} & & \\
\hline
\end{tabular}

All samples are prepared by weight in $0.2 \mathrm{M} \mathrm{NaCl}$ brine or deuterated brine. To the microemulsion, we add the hydrophobically modified poly(ethylene oxide) PEO$\mathrm{m}$ or PEO- $2 \mathrm{~m}$. The details on the components are indicated in Table 1. Samples are characterized by a volume fraction of nanodroplets $\Phi$ and by the number ' $r$ ' of $\mathrm{C}_{12}$ chains per nanodroplet. To calculate the number ' $\mathrm{r}$ ' of $\mathrm{C}_{12}$ chains per nanodroplet, we assume that the radius of the spherical nanodroplet does not change with increasing substitution of the surfactant by the copolymers. The precision of the value of ' $r$ ' depends on the accurate determination of the number of microemulsion nanodroplets per unit volume and thus on the determination of their size. All parameters necessary to calculate $\Phi$ from the sample composition are summarized in Table1. The samples prepared as described above, are thoroughly shaken to ensure homogenization and then kept at the temperature of observation, here $\mathrm{T}=25^{\circ} \mathrm{C}$, in a thermostated water bath for several days before visual examination. When a phase separation is observed, the samples are rehomogenized and set back to rest for a couple of days to confirm the observation. 


\section{Advanced Materials Letters www.vbripress.com/aml}

\section{SANS measurements}

Small angle neutron scattering (SANS) experiments are carried out on the PACE spectrometer at the LLB-Saclay (France). The configurations used were: $1.5 \mathrm{~m}$ at $6 \AA$ and $4.68 \mathrm{~m}$ at $13 \AA$. The range of scattering vectors covered is $0,004 \AA^{-1}<\mathrm{q}<0,16 \AA^{-1}$, the temperature $\mathrm{T}=25^{\circ} \mathrm{C}$. The scattering data are treated according to standard procedures. The differential scattering cross sections per unit sample volume denoted $I(q)=d \Sigma / d \Omega$ were put on absolute scale by using water as standard of known cross section. We obtain the intensity in absolute units $\left(\mathrm{cm}^{-1}\right)$ with accuracy better than $10 \%$. To simulate correctly the experimental spectra, all the model spectra are convoluted by the instrumental response function taking into account the uncertainty of the neutron wavelength and angular definition [33]. We carried out experiments with sphere contrast: the results obtained from samples prepared with hydrogenated constituents (decane, surfactant) in $\mathrm{D}_{2} \mathrm{O}$ - saline system $(0.2 \mathrm{~mol} / \mathrm{l})$. In this case we observe "homogeneous" nanodroplets on the scale of the spatial resolution of the scattering experiments $\pi / \mathrm{q}_{\max } \sim \pi / 0.21 \sim 15 \AA$. We calculate an average scattering length density of the microemulsion nanodroplets from scattering length density of the components using the data in Table 1. Thus, we calculate the contrast $\Delta \rho=6.810^{10} \mathrm{~cm}^{-2}$. In all samples studied here the scattering is essentially due to the nanodroplets, the scattering by the PEO chains being negligible due to their small mass as compared to the nanodroplets.

\section{Theoretical backgrounds}

\section{Form Factor $P(q)$}

SANS experiments provide information on the structure of colloidal solutions $[32,34]$, the scattered intensity I(q) by a dispersion of spherical colloids with a moderately spread distribution of size was modeled using the following expression [35],

$$
\mathrm{I}(\mathrm{q})=\Phi \cdot \mathrm{v} \cdot(\Delta \rho)^{2} \cdot \mathrm{P}(\mathrm{q}) \cdot \mathrm{S}(\mathrm{q})
$$

where $q\left(\AA^{-1}\right)$ is the scattering vector, $\Phi$ is the volume fraction of the aggregates, $\mathrm{v}\left(\mathrm{cm}^{3}\right)$ is the dry volume of the aggregates, $\Delta \rho$ is the scattering length density (corresponding to sphere contrast). $\mathrm{P}(\mathrm{q})$ is the form factor of the colloidal aggregates, and $\mathrm{P}(\mathrm{q} \rightarrow 0)=1$. The analysis of measurable structure factor $S(q)$ allows to extract information on the interparticle potential of the system (e.g., whether the interactions between particles are repulsive or attractive); it is unity at large or for very dilute samples where these interactions can be neglected. For monodisperse spheres of radius R [36],

$$
P(q, R)=\left[\frac{3[\operatorname{Sin}(q \cdot R)-(q \cdot R) \operatorname{Cos}(q \cdot R)]}{(q \cdot R)^{3}}\right]^{2}
$$

At high $\mathrm{q}$ values, the structure factor $\mathrm{S}(\mathrm{q} \rightarrow \infty)=1$, the scattered intensity is therefore only related to the form factor $\mathrm{P}(\mathrm{q})$. In this limit, we will use the Porod representation $\left(\mathrm{q}^{4} . \mathrm{I}(\mathrm{q})\right.$ ) as a function of ' $\mathrm{q}$ ' which amplifies the oscillations of $\mathrm{P}(\mathrm{q})$. Furthermore, one has to take into account of the polydispersity, we assume that the distribution size of spherical nanodroplets is described by a Gaussian distribution and write for the average form factor $P(q, \bar{R})$ :

$$
P(q, \bar{R})=\int_{0}^{+\infty} P(q, R) \cdot f(R) d R
$$

The function $f(R)$ is described by the Gauss distribution

$$
f(R)=\frac{1}{\sqrt{2 \pi} \Delta R} \exp \left[-\frac{(R-\bar{R})^{2}}{2 \Delta R^{2}}\right]
$$

with a mean radius $\bar{R}$ and a standard deviation $\Delta \mathrm{R}$.

\section{Structural and thermodynamic properties}

\section{a. Expression of the pair potential}

The bare microemulsion nanodroplets (reference case) are modeled by uniformly charged hard spheres, interacting by an effective potential $U(r)$, which is the sum of a hard sphere potential $\mathrm{U}_{\mathrm{HS}}(\mathrm{r})$, the attractive van der Waals $\mathrm{U}_{\mathrm{vdw}}(\mathrm{r})$ contribution and a screened Coulomb (Yukawatype) effective pair potential $\mathrm{U}_{\text {Coulomb }}(\mathrm{r})[\mathbf{3 7 , 3 8}]$,

$$
\mathrm{U}(\mathrm{r})=\mathrm{U}_{\mathrm{HS}}(\mathrm{r})+\mathrm{U}_{\mathrm{vdw}}(\mathrm{r})+\mathrm{U}_{\text {Coulomb }}(\mathrm{r}) \text { (4) }
$$

The pair potential $\mathrm{U}_{\mathrm{HS}}(\mathrm{r})$ between hard spheres of diameter $2 \mathrm{R}$ is given by:

$$
U_{H S}(r)=\left\{\begin{array}{ccc}
+\infty & \text { for } & r \leq 2 R \\
0 & \text { for } & r>2 R
\end{array}\right.
$$

Here, ' $r$ ' is the center-to-center distance and $\mathrm{R}$ is the radius of the nanodroplets.

The attractive interaction of van der Waals acts at distances between nanodroplets of the order of $10 \%$ of their diameter. In the case of two dispersed sphere, $\mathrm{U}_{\mathrm{vdw}}(\mathrm{r})$ potential is approximately [39],

$$
\mathrm{U}_{\mathrm{VdW}}(\mathrm{r})=-\frac{\mathrm{A}_{\mathrm{H}}}{6}\left(\frac{2 \mathrm{R}^{2}}{\mathrm{r}^{2}-4 \mathrm{R}^{2}}+\frac{2 \mathrm{R}^{2}}{\mathrm{r}^{2}}+\ln \left(\frac{\mathrm{r}^{2}-4 \mathrm{R}^{2}}{\mathrm{r}^{2}}\right)\right)(4 \mathrm{~b})
$$

$A_{H}$ is the effective Hamaker constant, $A_{H}$ is of the order of a few $k_{B} T[40]$. In this study, $A_{H}=1.1 k_{B} T$, this value is appropriate for decane droplets interacting through water [41].

In the Derjaguin-Landau-Verwey-Overbeek (DLVO) theory [42], the screened electrostatic repulsion (Yukawatype) between the charged colloidal spheres is described by the effective pair potential,

$$
\mathrm{U}_{\text {Coulomb }}(\mathrm{r})=\mathrm{k}_{\mathrm{B}} \mathrm{T} \frac{\mathrm{Z}_{\text {eff }}^{2} \mathrm{l}_{\mathrm{B}}}{(1+\kappa \mathrm{R})^{2}}
$$

The solvent is reduced to a structure less uniform continuum of dielectric constant $\varepsilon_{\mathrm{r}}=80$ and temperature $\mathrm{T}=25^{\circ} \mathrm{C}$ with a Bjerrum length of $1_{\mathrm{B}}=\mathrm{e}^{2} / 4 \varepsilon_{0} \varepsilon_{\mathrm{r}} \mathrm{k}_{\mathrm{B}} \mathrm{T}=7.18 \AA$. The Debye length determines 


\section{Advanced Materials Letters www.vbripress.com/aml}

the range of repulsive interactions $\mathrm{k}^{-1}$. In this study $\mathrm{k}^{-1}=6,77 \AA$, which corresponds to a concentration of small ions added to the solution, $Z_{\text {eff }}$ is the number of charge effective per nanodroplets [43], it determines the strength of the electrostatic repulsions.

To the bare microemulsion (reference case), we add telechelic polymers with one and two ends (PEO-m, PEO$2 \mathrm{~m}$ ). The addition of PEO-m induces a steric repulsive potential between nanodroplets due to the resistance to the confinement. To calculate $\mathrm{S}(\mathrm{q})$ for this situation, an additional repulsive contribution due to the decoration of the nanodroplets by the chains of PEO was added to the existing interaction in the bare microemulsion.

$$
\mathrm{U}_{1}(\mathrm{r})=\mathrm{U}(\mathrm{r})+\mathrm{V}(\mathrm{r})
$$

The potential $\mathrm{V}(\mathrm{r})$ is a steric repulsive interaction that is described here by a Yukawa-type potential added to the previous one defined in eq. (4). We have:

$$
\mathrm{V}(\mathrm{r})=\mathrm{V}_{\text {steric }}\left(\frac{2 \mathrm{R}}{\mathrm{r}}\right) \exp \left(-\frac{\mathrm{r}-2 \mathrm{R}}{\lambda_{\text {steric }}}\right)
$$

where $\mathrm{V}_{\text {steric }}$ describes the strength of the potential via its value at contact and $\lambda_{\text {steric }}$ its characteristic range.

The last case study is to add the PEO-2m with two ends to the bare microemulsion. To calculate $S(q)$, the repulsive effect is always present and an interesting additional contribution due to the bridging of nanodroplets by PEO chains must be added to the existing interaction in the bare microemulsion,

$$
\mathrm{U}_{2}(\mathrm{r})=\mathrm{U}(\mathrm{r})+\mathrm{V}(\mathrm{r})+\mathrm{W}(\mathrm{r})
$$

The potential $\mathrm{W}(\mathrm{r})$ is an effective attractive interaction potential of entropic origin [12].

We propose here a simple model of Yukawa attractive potential,

$$
\mathrm{W}(\mathrm{r})=\mathrm{W}_{\text {bridging }}\left(\frac{2 \mathrm{R}}{\mathrm{r}}\right) \exp \left(-\frac{\mathrm{r}-2 \mathrm{R}}{\lambda_{\text {bridging }}}\right)
$$

where $\mathrm{W}_{\text {bridging }}$ is an attractive contact potential and $\lambda_{\text {bridging }}$ its characteristic range.

The range of interactions $\lambda_{\text {steric }}$ and $\lambda_{\text {bridging }}$ of the polymers is set by their size. In the numerical study, we have fixed these parameters: $\lambda_{\text {steric }}$ is approximately $35 \AA$ which is close to the radius of gyration of PEO-m chain with molar mass $\mathrm{M}\left(\mathrm{R}_{\mathrm{g}}=0.107 \pm 0.1 \mathrm{M}^{0.63 \pm 0.01} \AA\right)$ $[\mathbf{4 4 , 4 5}]$. In the same way, $\lambda_{\text {bridging }}$ is of the order of $100 \AA$ corresponding to the average end-to-end distance of PEO- $2 \mathrm{~m}$ chain, i.e., three times the radius of gyration [24].

\section{b. Structure factor}

The validity of the potential $U(r)$ is tested by comparing the experimental and numerical structure factor $S(q)$. To calculate $\mathrm{S}(\mathrm{q})$ which is the Fourier transform of the pair correlation function $\mathrm{g}(\mathrm{r})$, we use a program developed by Belloni based on the Ornstein-Zernike integral and hypernetted-chain approximation (HNC) closure relation. For more details, consult the appendix.

\section{c. The excess of internal energy}

The pair correlation function $g(r)$ and the pair potential $\mathrm{U}(\mathrm{r})$ provide access to the thermodynamic properties of liquids. Thus, the relationship giving the internal energy:

$$
\begin{aligned}
& \mathrm{E}=\mathrm{E}_{\text {kin }}+\mathrm{U}\left(\mathrm{r}^{\mathrm{N}}\right) \\
& \mathrm{E}=\frac{3}{2} \mathrm{NK}_{\mathrm{B}} \mathrm{T}+\frac{1}{2} \rho \mathrm{N} \int 4 \pi \mathrm{r}^{2} \mathrm{U}(\mathrm{r}) \mathrm{g}(\mathrm{r}) \mathrm{dr}
\end{aligned}
$$

The internal energy is the sum of a kinetic part, $\left(\frac{3}{2} \mathrm{NK}_{\mathrm{B}} \mathrm{T}\right)$ and an interaction part, $\mathrm{U}\left(\mathrm{r}^{\mathrm{N}}\right)$.

The excess of internal energy is given by,

$$
\Delta \mathrm{E}=\mathrm{E}-\mathrm{E}_{\text {kin }}=\mathrm{U}\left(\mathrm{r}^{\mathrm{N}}\right)=\frac{1}{2} \rho \mathrm{N} \int 4 \pi \mathrm{r}^{2} \mathrm{U}(\mathrm{r}) \mathrm{g}(\mathrm{r}) \mathrm{dr}
$$

The sign of $\Delta \mathrm{E}$ indicates the nature of the interaction, repulsive for $\Delta \mathrm{E}\rangle 0$ and attractive for $\Delta \mathrm{E}\langle 0$.

\section{Results and discussion}

\section{Shape and size of microemulsion nanodroplets}

We have studied the SANS patterns for bare microemulsion as reference case and for samples with POE-m or POE-2m at different values of $\Phi$ and $r$ (number of $\mathrm{C}_{12}$-stickers per nanodroplet). We have showed that the spherical shape of microemulsion nanodroplets is identical from $\Phi=1.4 \%$ to $\Phi=26.5 \%$, and in the presence of PEO$\mathrm{m}$ or PEO- $2 \mathrm{~m}$ at different $r$, with an average radius of $62 \AA$, and $\Delta \mathrm{R}=6.2 \AA$, i.e. a polydispersity $\mathrm{p}=10 \%$. This indicates that the droplet structure of the microemulsion remains unaltered by the polymer addition $[\mathbf{4 3}]$.

\section{Structural properties}

The validity of the steric and bridging potential caused by the presence of PEO-m or PEO- $2 \mathrm{~m}$ is tested by comparing the experimental and numerical structure factor $\mathrm{S}(\mathrm{q})$, whichis the Fourier transform of the pair correlation function $\mathrm{g}(\mathrm{r})$. The pair correlation function is calculated with a program kindly provided by Luc Belloni. In this program, the $(\mathrm{OZ})$ integral equation associated with the (HNC) is used, with an effective pair potential for each experimental situation. It should be noted that in the calculation of $S(q), Z_{\text {eff }}$ is the only adjustable parameter for the bare microemulsion, $\mathrm{V}_{\text {steric }}$ and $\mathrm{W}_{\text {bridging }}$ are the only adjustable parameters for the microemulsion with POE-m or POE-2m. For each fraction studied, experimental structure factor is obtained by dividing scattered intensity I(q) by the form factor of the bare microemulsion at very low volume fraction $\left(\mathrm{S}(\mathrm{q})=\frac{\mathrm{I}(\mathrm{q}, \Phi)}{\mathrm{I}(\mathrm{q}, \Phi=1.4 \%)}\right)$.

\section{a. Reference case: Bare microemulsion}

Fig. 1 represents a comparison of experimental and numerical structures factors at different $\Phi$ (from $2.8 \%$ to $26.5 \%$ ). Generally, an excellent qualitative agreement is observed, proving that the model of the proposed potential is satisfactory in the range of the wave vectors and for all fractions studied.The peak location carries information 


\section{Advanced Materials Letters www.vbripress.com/aml}

about the mean inter-nanodroplet distance, and the sharpness of the peak about the fluctuations around this mean distance, which in turn is dictated by the nanodroplet interaction potential. At low volume fraction, $\Phi=2.8 \%$ and $\Phi=6.98 \%$, the structure factor $S(q)$ shows a weak and broad principal peak. This is a signature of a weak repulsive interaction between nanodroplets, the suspension is less ordered and nanodroplets have more positional fluctuations. At high volume fraction $\Phi=14 \%$, $26.5 \%$, we observe a pronounced and narrow principal peak and apparition of the second peak, which reflect the existence of strong repulsions, the suspension is more ordered and the nanodroplets do not fluctuate much around their average positions.

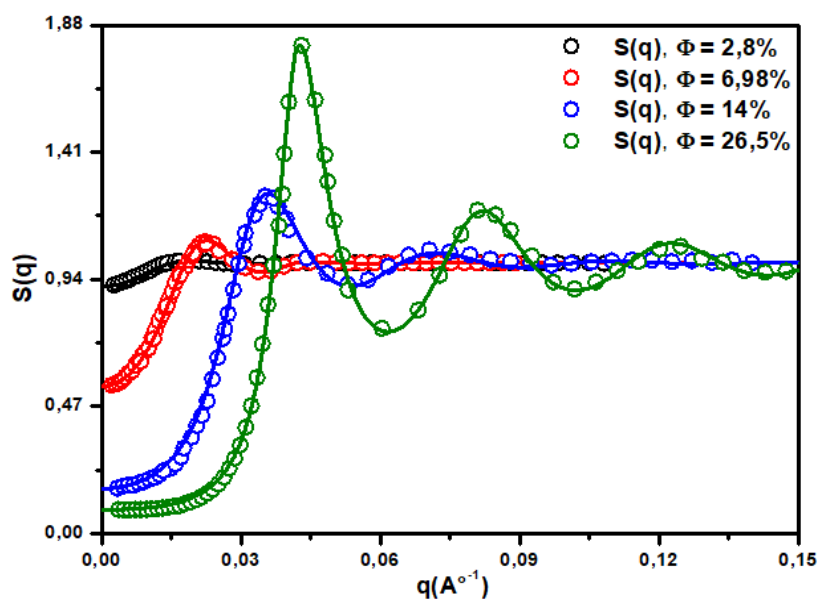

Fig. 1. Comparison of structure factorS(q), for bare microemulsion, at different $\Phi(-2.8 \%,-6.98 \%,-14 \%,-26.5 \%)$.

A general remark, as expected, when the volume fraction increases from $2.8 \%$ to $26.5 \%$, the height of the (principal) peak of the structure factor becomes more pronounced (from 1.015 to 1.83). This change in amplitude is accompanied by a shift toward in high values of $q$. As the position of the correlation peak indicates the average distance between nanodroplets, $(q \sim 2 \pi / d)$, the preferred distance is estimated at different volume fraction $(\Phi=2.8 \%: \mathrm{d} \sim 328 \AA, \Phi=6.98 \%: \mathrm{d} \sim 279 \AA, \Phi=14 \%$ : $\mathrm{d} \sim 175 \AA, \Phi=26.5 \%: \mathrm{d} \sim 147 \AA$ ); we note that it decreases with increasing $\Phi$ as $\mathrm{d} \sim \Phi^{-1 / 3}$, all results are summarized in Table 2.

We followed the evolution of the interaction between nanodroplet, we consider the colloidal particles are described as uniformly charged hard spheres interacting by an effective potential, which is the sum of a van der Waals attractive term, a hard sphere repulsive potential and screened Coulombic repulsive potential. In Fig.1, the solid line is the spectra calculated with the parameters: $\bar{R}=62 \AA, \Delta \mathrm{R}=6.2 \AA, \mathrm{T}=298^{\circ} \mathrm{K}, \Delta \rho=6,8.10^{10} \mathrm{~cm}^{-2}, \mathrm{k}^{-1}=$ $6,77 \AA, A_{H}=1.1 k_{B} T . Z_{\text {eff }}=130$ is the only adjustable parameter.

In summary, the droplets of the bare microemulsion are well represented by spherical shapes interacting through a potential that is the sum of an attractive and repulsive contribution. Now, we are interested in the features of the effective interactions introduced upon addition of polymers POE-m and POE- $2 \mathrm{~m}$ to the bare microemulsion.

Table 2. Analysis of S(q) curves obtained by SANS and HNC.

\begin{tabular}{cccc}
\hline Method & $\boldsymbol{\Phi}$ & $\mathbf{S}_{\max }$ & $\mathbf{D}(\mathbf{\AA})$ \\
\hline SANS & $2.8 \%$ & 1.012 & 328 \\
HNC & & 1.009 & 330 \\
SANS & $6.98 \%$ & 1.087 & 279 \\
HNC & & 1.083 & 278 \\
SANS & $14 \%$ & 1.247 & 175 \\
HNC & & 1.247 & 177 \\
SANS & $26.5 \%$ & 1.833 & 147 \\
HNC & & 1.85 & 147
\end{tabular}

\section{b. Microemulsion with PEO-m}

In Fig. 2, we report the structure factor $\mathrm{S}(\mathrm{q})$ of the microemulsion with PEO-m $(r=4 \mathrm{~m}, 8 \mathrm{~m}, 12 \mathrm{~m})$, for a low and high nanodroplet volume fraction. At low volume fraction, $\Phi=6.98 \%$ (Fig.2a), firstly, we note the diminution of $S(q)$ at very low q's when ' $r$ ' varies from 0 to 12 , secondly, the peak becomes slightly more pronounced ( $S_{\max }$ varies from 1.06 to 1.16$)$ induced by the decoration of the droplet by POE-m. But the position of the maximum of the correlation peak remains at the same position with addition of POE-m (see Table 3). At high volume fraction $\Phi=26.5 \%$ (Fig.2b), the principal peakheight becomes more pronounced (from 1.86 to 2.1) when $r$ varies from 4 to 12 , see Table 3. As expected, the addition of POE-m does not influence on the position of the correlation peak (distance between the nanodroplets remains approximately constant). Such results indicate that the addition of POE-m to the bare microemulsion introduces an effective steric repulsive interaction. This interaction is repulsive at distance shorter than polymer size due to the resistance of the confinement.

Now, our objective is to "weight" the steric repulsion due to the presence of PEO-m for all volume fraction $\Phi$.In Fig.2, the solid line is the spectra calculated with the parameters: $\bar{R}=62 \AA, \Delta \mathrm{R}=6.2 \AA, \mathrm{T}=298^{\circ} \mathrm{K}, \mathrm{Z}_{\mathrm{eff}}=130$, $\Delta \rho=6,8 \cdot 10^{10} \mathrm{~cm}^{-2}, \mathrm{~A}_{\mathrm{H}}=1.1 \mathrm{k}_{\mathrm{B}} \mathrm{T}, \mathrm{k}^{-1}=6,77 \AA$.

The range of steric interactions $\lambda_{\text {steric }}$ of the polymers PEO-m is fixed at $35 \AA, V_{\text {steric }}$ is the only adjustable parameter. The fits reproduce in a satisfying manner the low-q depression of the structure factor. For PEO$\mathrm{m}(\mathrm{r}=4 \mathrm{~m})$, the best numerical fit is obtained with a steric potential in contact, $\mathrm{V}_{\text {steric }}=2.6 \mathrm{k}_{\mathrm{B}} \mathrm{T}$. When we doubled the amount of PEO-m added $(\mathrm{r}=8 \mathrm{~m})$, the potential in contcact $\mathrm{V}_{\text {steric }}=5.2 \mathrm{k}_{\mathrm{B}} \mathrm{T}$. In the same way, the amount of PEO-m added $(\mathrm{r}=12 \mathrm{~m})$ gives a potential in contact $\mathrm{V}_{\text {steric }}=7.8 \mathrm{k}_{\mathrm{B}} \mathrm{T}$ (Table 3).

To summarize, the steric potential in contact, $\mathrm{V}_{\text {steric }}$, increases roughly proportionnaly to the amount of polymer added, the proportionality factor $\mathrm{V}_{\text {steric }} / \mathrm{r}$ is 0.65 . This is similar to what has been found in the litterature with other microemulsion systems decorated with hydrophobically modified polymers [23]. 

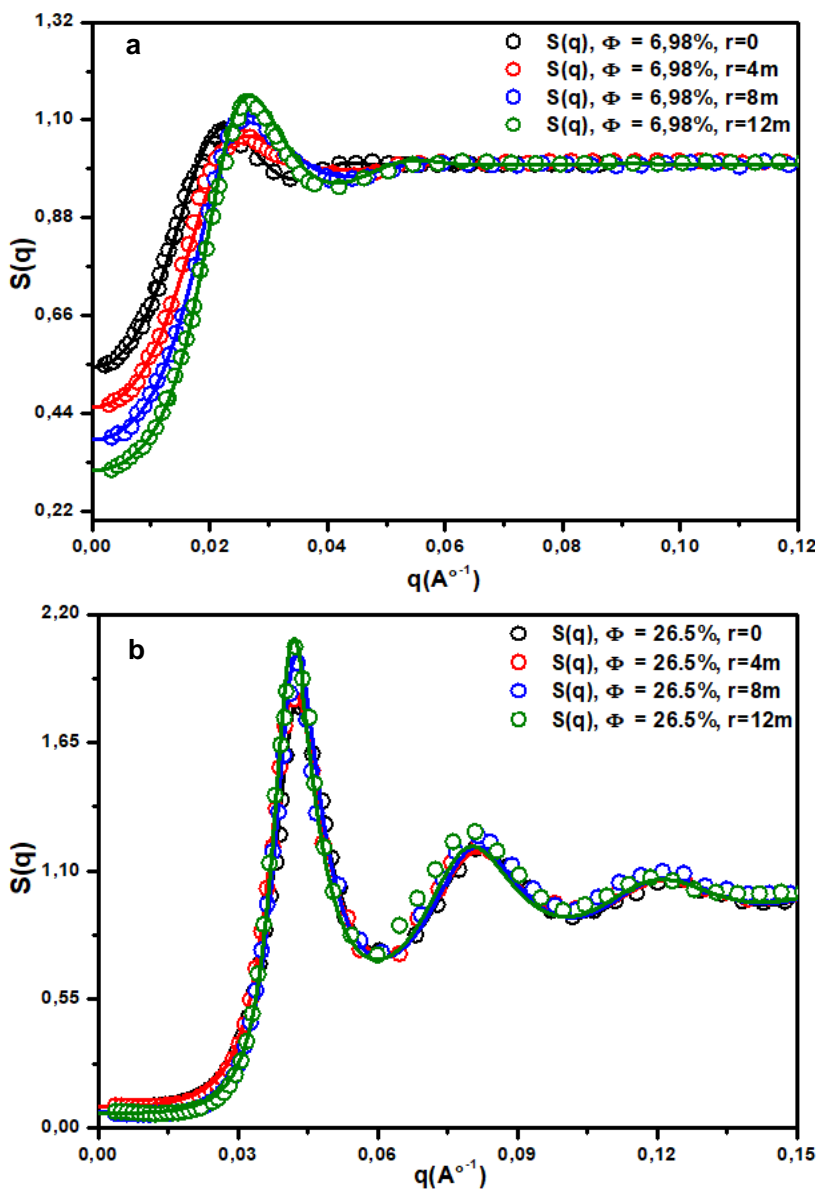

Fig. 2. Comparison of structure factor $\mathrm{S}(\mathrm{q})$, for bare microemulsion $(\circ \mathrm{r}=0)$ and with PEO-m $(\circ \mathrm{r}=4 \mathrm{~m}, \circ \mathrm{r}=8 \mathrm{~m}, \circ \mathrm{r}=12 \mathrm{~m})$, in the diluted and concentrated cases $(\Phi=6.98 \%, \Phi=26.5 \%)$.

Table 3. Comparison of results for: $S(0), S_{\max }$, distance between nanodroplets (d), $\mathrm{V}_{\text {steric }}, \mathrm{W}_{\text {bridging, for bare microemulsion, with PEO-m }}$ $(4 \mathrm{~m}, 8 \mathrm{~m}, 12 \mathrm{~m})$ and PEO-2m $\left(4 \_2 \mathrm{~m}, 8 \_2 \mathrm{~m}\right)$, in diluted $(\Phi=6.98 \%)$ and concentrated case $(\Phi=26.5 \%)$.

\begin{tabular}{|c|c|c|c|c|c|c|c|c|c|c|}
\hline \multirow{2}{*}{$\begin{array}{l}\text { Cases } \\
\text { Studies }\end{array}$} & \multicolumn{5}{|c|}{$\Phi=6.98 \%$} & \multicolumn{5}{|c|}{$\Phi=26.5 \%$} \\
\hline & $S(0)$ & $S_{\max }$ & $\mathbf{d}(\mathbf{A})$ & $\begin{array}{l}V_{\text {steric }} \\
(\mathrm{kBT})\end{array}$ & $\begin{array}{c}\text { Wbridging } \\
\text { (kBT) }\end{array}$ & $\mathbf{S}(\mathbf{0})$ & $S_{\max }$ & $d(\AA)$ & $\begin{array}{l}V_{\text {steric }} \\
(\mathbf{k B T})\end{array}$ & $\begin{array}{l}\text { Wbridging } \\
(\mathbf{k B T})\end{array}$ \\
\hline$r=0$ & 0,55 & 1.083 & 279 & - & - & 0,088 & 1.8 & 147 & - & - \\
\hline $\mathrm{r}=4 \mathrm{~m}$ & 0,45 & 1,064 & 238 & 2.6 & - & 0,09 & 1,86 & 149 & 2.6 & - \\
\hline $\mathrm{r}=8 \mathrm{~m}$ & 0,38 & 1,11 & 235 & 5.2 & - & 0,062 & 2,03 & 148 & 5.2 & - \\
\hline$r=12 m$ & 0,31 & 1,156 & 235 & 7.8 & - & 0,06 & 2,1 & 149 & 7.8 & - \\
\hline $\mathrm{r}=4 \_2 \mathrm{~m}$ & 0,6 & 1,085 & 216 & 2.6 & -0.53 & 0,05 & 1,9 & 149 & 2.6 & -0.53 \\
\hline $\mathrm{r}=8 \_2 \mathrm{~m}$ & 0.62 & 1,074 & 223 & 5.2 & -1.05 & 0,03 & 2,02 & 149 & 5.2 & -1.05 \\
\hline
\end{tabular}

\section{c. Microemulsion with PEO-2m}

In this section, we want to understand the effect of the addition of PEO- $2 \mathrm{~m}$ on the interactions between nanodroplets for a low and high nanodroplet volume fraction $(\Phi=6.98 \%, 26.5 \%)$. At the low volume fraction $\Phi=6.98 \%$ (Fig. 3a), we compare the structure factor of the bare microemulsion $(\mathrm{r}=0)$ with PEO-2m (4_2m, $\left.8 \_2 \mathrm{~m}\right)$. We note a large rise of $\mathrm{S}(\mathrm{q})$ at very low q's as $\mathrm{r}$ increases from 0 to 8 . This indicates that addition of the POE- $2 \mathrm{~m}$ to the bare microemulsion introduces an effective attractive interaction between the nanodroplets. The attractive interaction can be pictured as due to the longer time the droplets spend close to one another in the low volume fraction because of the bridging which leads to more accessible configurations for the PEO chain and thus to an increased entropy. The structure factor gives us another clue, namely, the distance between the droplets exists in the sample, as indicated by the existence of a correlation peak. The shoulder is shifted to its smaller values of inter-distance between nanodroplets $(\mathrm{d} \sim 279 \AA)$, for bare microemulsion $(\mathrm{r}=0), \mathrm{d} \sim 216 \AA$, for microemulsion with PEO-2m $\left(\mathrm{r}=4 \_2 \mathrm{~m}\right)$, (see Table 3 ). The distance $(\mathrm{d} \sim 226 \AA)$ is shorter than the average distance expected between nanodroplets at $\Phi=6.98 \%$ $\left(\mathrm{d}: \Phi^{-1 / 3}\right)$. This distance is imposed by the telechelic polymers in configurations corresponding to their average end-to-end distance $(\sim 100 \AA)$ [20]. The centre-to-centre distance of two adjacent nanodroplets in an aggregate is then $\sim 224 \AA$, in excellent agreement with the experimental observation. At high volume fraction $(\Phi=26.5 \%)$ (Fig. $3 \mathbf{b})$, the correlation peak of $\mathrm{S}(\mathrm{q})$ is very narrow and the position of nanodroplets is more localized. The height of peak remains constant $\left(S_{\max } \sim 2\right)$ when $r$ varies from 4 to 8 . The preferred distance varies as $\Phi^{-1 / 3}$ (Table 3).

Another aspect of this study is the "weighting" of the attractive potential due to the presence of the polymer chain PEO-2m for all volume fraction $\Phi$ (Fig. 3a, Fig. 3b). An excellent agreement between experimental and numerical spectra is observed.The fits reproduce in a satisfying manner the low-q depression of the structure factor. At low volume fraction $(\Phi=6.98 \%)$ (Fig.3a), the best numerical fit is obtained with following parameters:

- For $\left(r=4 \_2 \mathrm{~m}\right): \mathrm{V}_{\text {steric }}=2.6 \mathrm{k}_{\mathrm{B}} \mathrm{T}, \mathrm{W}_{\text {bridging }}=-0.53 \mathrm{k}_{\mathrm{B}} \mathrm{T}$.

- For $\left(\mathrm{r}=8 \_2 \mathrm{~m}\right): \mathrm{V}_{\text {steric }}=5.2 \mathrm{k}_{\mathrm{B}} \mathrm{T}, \mathrm{W}_{\text {bridging }}=-1.05 \mathrm{k}_{\mathrm{B}} \mathrm{T}$.

We note that the prefactor $\mathrm{W}_{\text {bridging }}$ increases again linearly with $\mathrm{r}$ ( $\mathrm{W}_{\text {bridging }} / \mathrm{r}=0.13$ ). This is expected from the theory by Bathia and Russel [20], or Elghazrani et. al.,[24]. At high volume fraction $(\Phi=26.5 \%)$ (Fig. 4c), it is observed that all the spectra are superimposable, the difference in behavior between the two polymers POE-m and POE- $2 \mathrm{~m}$ gradually fades by increasing $\Phi$, i.e., the addition of the polymer chains PEO- $2 \mathrm{~m}$ has the same effect as POE-m on the microemulsion. The droplets are already closer than the typical attractive interaction range, the distance between nanodroplets becomes equal to or smaller than the end-to-end distance of polymer chain, bridging can occur without adding any attractive component to the interaction between nanodroplets and the repulsive interaction part plays a dominant role. Nevertheless, some influence of the bridge persists (Fig.3b). The attractive interaction exists but it is lower. For the sample PEO-2m(r=4_2m), the best numerical fit is obtained with the range of the bridging interaction, $\lambda_{\text {bridging }}=100 \AA, \mathrm{V}_{\text {steric }}=2.6 \mathrm{k}_{\mathrm{B}} \mathrm{T}, \mathrm{W}_{\text {bridging }}=-0.53 \mathrm{k}_{\mathrm{B}} \mathrm{T}(\mathrm{See}$ Table 3). 

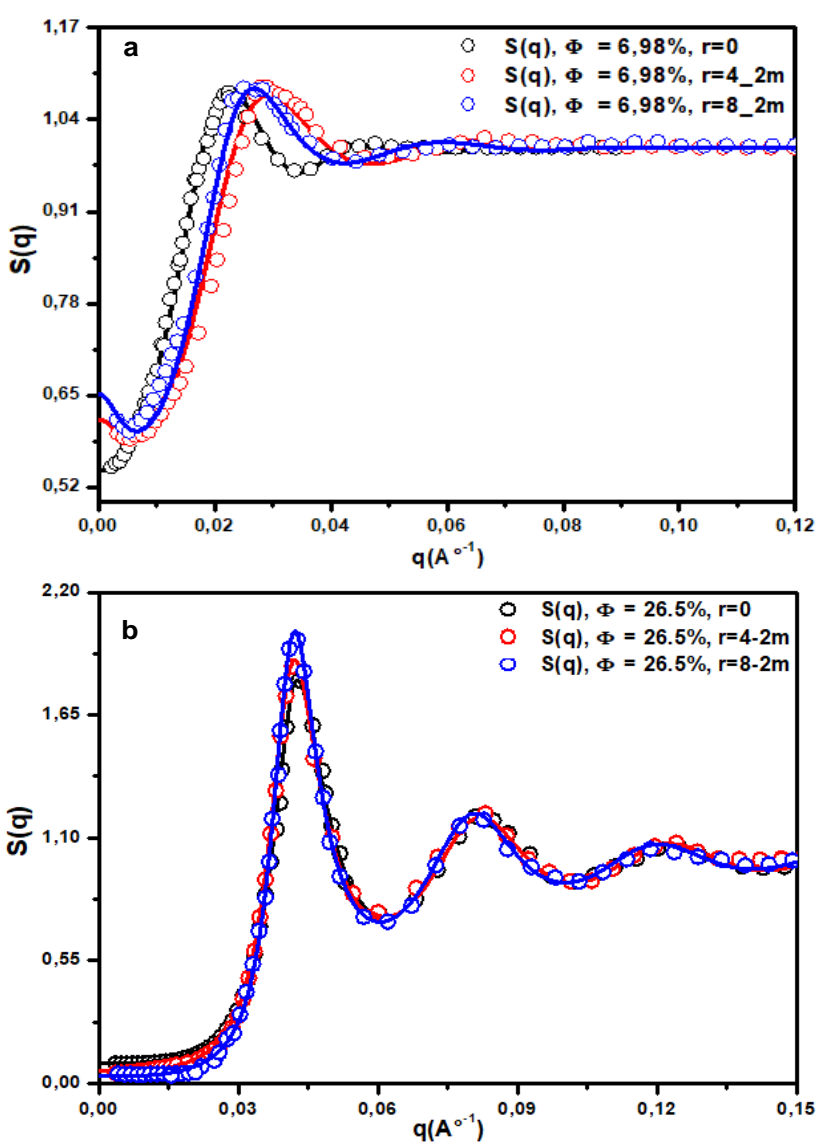

Fig. 3. Comparison of structure factor $\mathrm{S}(\mathrm{q})$, for bare microemulsion $(\circ \mathrm{r}=0)$ and with PEO-2m $\left(\circ \mathrm{r}=4 \_2 \mathrm{~m}, \circ \mathrm{r}=8 \_2 \mathrm{~m}\right)$, in the diluted and concentrated cases $(\Phi=6.98 \%, \Phi=26.5 \%)$.

In Fig.3, the solid line is the spectra calculated with the parameters: $\bar{R}=62 \AA, \Delta \mathrm{R}=6.2 \AA, \mathrm{T}=298^{\circ} \mathrm{K}, \mathrm{A}_{\mathrm{H}}=$ $1.1 \mathrm{k}_{\mathrm{B}} \mathrm{T}, \Delta \rho=6,8.10^{10} \mathrm{~cm}^{-2}, \mathrm{Z}_{\text {eff }}=130, \mathrm{k}^{-1}=6,77 \AA$, $\lambda_{\text {steric }}=35 \AA$ and $\lambda_{\text {bridging }}=100 \AA$ :

- For $\left(\mathrm{r}=4 \_2 \mathrm{~m}\right): \mathrm{V}_{\text {steric }}=2.6 \mathrm{k}_{\mathrm{B}} \mathrm{T}, \mathrm{W}_{\text {bridging }}=-0.53 \mathrm{k}_{\mathrm{B}} \mathrm{T}$

- $\quad$ For $\left(\mathrm{r}=8 \_2 \mathrm{~m}\right)$ : $\mathrm{V}_{\text {steric }}=5.2 \mathrm{k}_{\mathrm{B}} \mathrm{T}, \mathrm{W}_{\text {bridging }}=-1.05 \mathrm{k}_{\mathrm{B}} \mathrm{T}$

In summary, in diluted case, the bridging polymer chains introduce an effective attraction interaction between nanodroplets. While in concentrated case, PEO$2 \mathrm{~m}$ will add to the repulsion in the same way as the decorating polymer chains PEO-m resisting interpenetration and longing to swell in brine. Therefore, both polymers have the same effect on the microemulsion; the reinforcement of the repulsive potential.

\section{Thermodynamical properties}

The aim of this section is to verify through the thermodynamic properties (internal energy) the results found in the previous section.

Generally, the stability of the colloidal suspensions studied by the integral equations is discussed in terms of the internal energy, taken as a thermodynamic potential, instead of the free energy. The sign of $\Delta \mathrm{E}$ (internal energy excess (Eq.7a) indicates the nature of the interaction repulsive for $\Delta \mathrm{E}\rangle 0$ and attractive for $\Delta \mathrm{E}\langle 0$.
Fig. 4a shows the variation of $\Delta \mathrm{E} / \mathrm{Nk}_{\mathrm{B}} \mathrm{T}$ with volume fraction $\Phi$, for various values of $\mathrm{r}(0,4 \mathrm{~m}, 8 \mathrm{~m}, 12 \mathrm{~m})$. We observe a monotonic increase of the excessive internal energy with increasing $\Phi$ or polymer concentration. This result is in agreement with the behaviours of the repulsive interaction between nanodroplets and the compressibility [44]. Fig. 4b indicates the evolution of internal energy excess with volume fraction $\Phi$ for various values of $\mathrm{r}(0$, 4_2m, 8_2m). Remark that the internal energy excess presents two volume fraction-regimes. The first corresponds to low volume fraction, $\Delta \mathrm{E} / \mathrm{Nk}_{\mathrm{B}} \mathrm{T}$ takes negative values which are sensitive to the variation of the number of $\mathrm{C}_{12}$ chains. Hence, by increasing the amount of the POE- $2 \mathrm{~m}$ added, attractive effect is reinforced. The second corresponds to higher volume fraction, $\Delta \mathrm{E} / \mathrm{Nk}_{\mathrm{B}} \mathrm{T}$ takes positive values. Consequently, the bridging polymer chains will add to the repulsion in the same way as the decorating polymer chains, i.e. the reinforcement of the repulsion. In Table 4, we report the results of $\Delta \mathrm{E} / \mathrm{Nk}_{\mathrm{B}} \mathrm{T}$, found for all fractions studied (from $1 \%$ to $30 \%$ ).
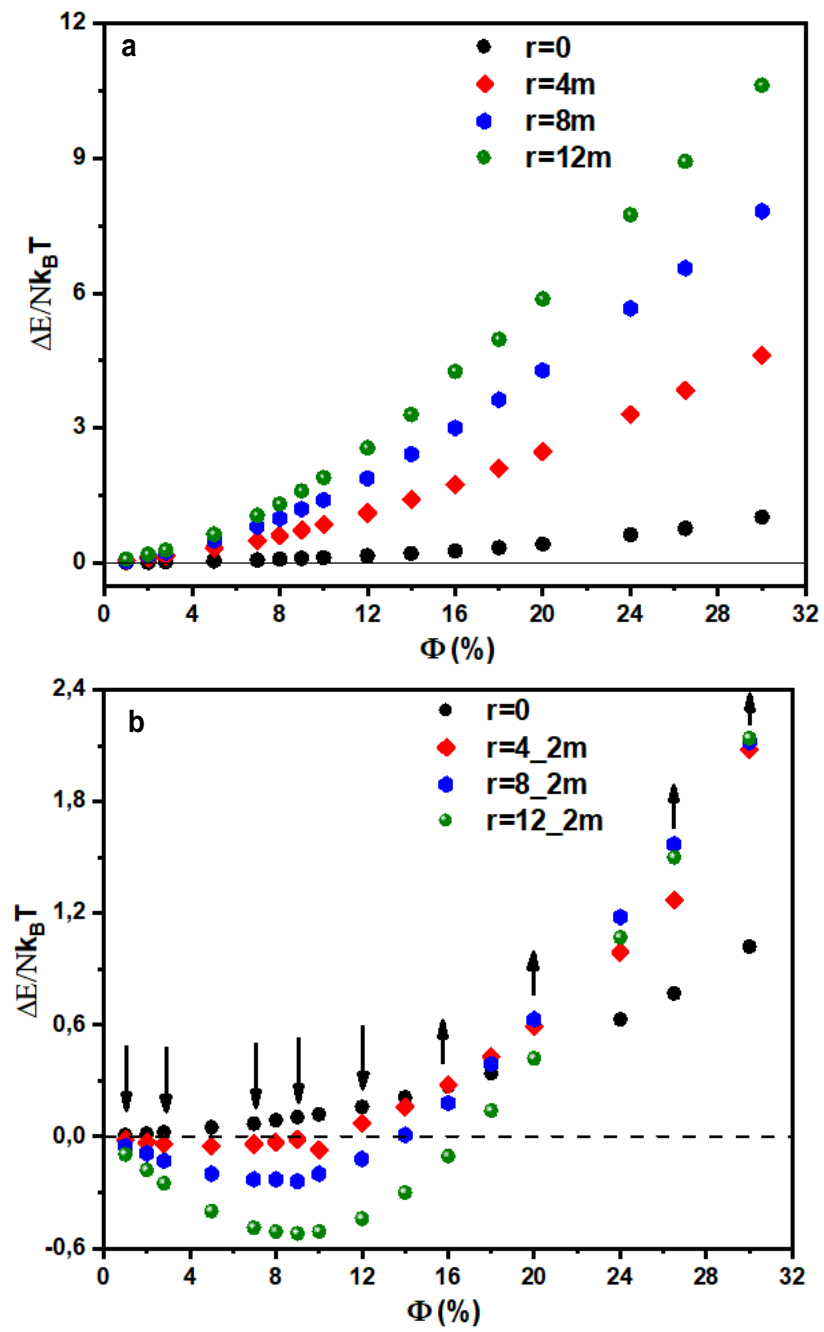

Fig. 4. Dimensionless excess internal energy, $\Delta \mathrm{E} / \mathrm{Nk}_{\mathrm{B}}$ Tversus the volume fraction $\Phi$, for bare microemulsion $(\circ \mathrm{r}=0)$, with PEO-m $(\circ \mathrm{r}=4 \mathrm{~m}, \circ \mathrm{r}=8 \mathrm{~m}, \circ \mathrm{r}=12 \mathrm{~m})$ and PEO-2m $\left(\circ \mathrm{r}=4 \_2 \mathrm{~m}, \circ \mathrm{r}=8 \_2 \mathrm{~m}\right.$, $\left.\circ \mathrm{r}=12 \_2 \mathrm{~m}\right)$. 
Table 4. Dimensionless excess internal energy, $\Delta E / \mathrm{Nk}_{B} T$ of the bare microemulsion, with PEO-m and PEO-2m, at different volume fractions $\Phi$ (from $1 \%$ to $30 \%$ ).

\begin{tabular}{c|c|c|c|c|c|c}
\hline $\begin{array}{c}\mathbf{\Delta \mathbf { E }} \\
\boldsymbol{\Phi}(\boldsymbol{\%} \mathbf{0})\end{array}$ & $\mathbf{r}=\mathbf{0}$ & $\mathbf{r}=\mathbf{4 m}$ & $\mathbf{r}=\mathbf{8 m}$ & $\mathbf{r}=\mathbf{1 2 m}$ & $\mathbf{r}=\mathbf{4} \_\mathbf{2 m}$ & $\mathbf{r}=\mathbf{8} \_\mathbf{2 m}$ \\
\hline 2,8 & 0,024 & 0,16 & 0,24 & 0,29 & $-0,04$ & $-0,13$ \\
6,98 & 0,07 & 0,5 & 0,81 & 1,06 & $-0,04$ & $-0,23$ \\
14 & 0,21 & 1,41 & 2,42 & 3,3 & 0,16 & 0,009 \\
26,5 & 0,77 & 3,84 & 6,55 & 8,93 & 1,27 & 1,57 \\
\hline
\end{tabular}

\section{Conclusion \& future prospective}

In this paper, we studied the thermodynamic and structural properties of positively charged $\mathrm{O} / \mathrm{W}$ microemulsion spherical nanodroplets suspended in salt water and decorated with telechelic polymers PEO-m and PEO-2m by using SANS. We have shown that microemulsion nanodroplets do not change shape and size, by varying the volume fraction, and in the presence of polymers with different quantities. They are described by slightly polydispersed spheres with an average radius of $62 \AA(\sigma=$ $10 \%)$. The structure factor can be reproduced qualitatively using the (OZ) equation, with the HNC approximation. The system is described as uniformly charged hard spheres. We have adopted the Yukawa interaction potential between nanodroplets. The addition of PEO-m with different amounts induces repulsive interactions between nanodroplets. At low and high volume fraction, the repulsive potential increases linearly with the amount of polymer added. The addition of PEO- $2 \mathrm{~m}$ to the bare microemulsion leads to an effective attractive bridging interaction between the nanodroplets in the diluted case. In the concentrated case, the polymers have the same effect on the microemulsion; the reinforcement of the repulsive potential. It must be stressed that the model potentials for the effective interaction introduced by the polymers allowed us to have a reasonable agreement between the calculated and experimental spectra for all volume fractions studied. The dynamical properties of the system will be described by using the molecular dynamics simulation in a forthcoming paper.

\section{Acknowledgements}

This work was achieved in collaboration with the Laboratory of Montpellier (Charles Coulomb). We are much indebted to Professors G. Porte and J. Appell, for their assistance and their support during the realization for this work, R. Aznar, for synthesizing the copolymer used in this work, and L. Auvray and D. Lairez, for their useful help during SANS experiments performed on line PACE at Leon Brillouin Laboratory. We are also grateful to Professor L. Belloni who gave us the code for the computation of the structure factor and for the fruitful discussions which enabled us the determination of effective charge.

\section{Conflicts of interest}

The authors declare that they have no conflict of interest.

\section{Supporting information}

The theoretical methods which allow to calculate the $\mathrm{g}(\mathrm{r})$ are based on the Ornstein-Zernike $(\mathrm{OZ})$ integral equation,

$$
\mathrm{h}(\overrightarrow{\mathrm{r}})=\mathrm{c}(\overrightarrow{\mathrm{r}})+\rho \int \mathrm{c}\left(\left|\overrightarrow{\mathrm{r}}-\overrightarrow{\mathrm{r}^{\prime}}\right|\right) \mathrm{h}\left(\overrightarrow{\mathrm{r}^{\prime}}\right) \mathrm{dr}
$$

The $(\mathrm{OZ})$ equation is a relation between the total correlation function $\mathrm{h}(\overrightarrow{\mathrm{r}})=\mathrm{g}(\overrightarrow{\mathrm{r}})-1$, and the direct correlation function $\mathrm{c}(\overrightarrow{\mathrm{r}})[\mathbf{4 6}, \mathbf{4 7}]$.

To compute $\mathrm{g}(\mathrm{r})$, the $(\mathrm{OZ})$ equation was solved using the hypernetted-chain approximation (HNC) [48]. In terms of $\mathrm{c}(\mathrm{r})$, the (HNC) closure relation,

$$
\mathrm{c}(\mathrm{r}) \approx-\beta \mathrm{U}(\mathrm{r})+\mathrm{h}(\mathrm{r})-\ln [1+\mathrm{h}(\mathrm{r})]
$$

Exponentiation gives,

$$
\mathrm{g}(\mathrm{r}) \approx \exp [-\beta \mathrm{U}(\mathrm{r})+\mathrm{h}(\mathrm{r})-\mathrm{c}(\mathrm{r})]
$$

The interactions are very weak in the very diluted sample $(\Phi=1.4 \%)$. This is the reason why, we have chosen to divide the scattered intensities I (q) of all the samples by I (q, $\Phi=1.4 \%$ ), see the Figure below.

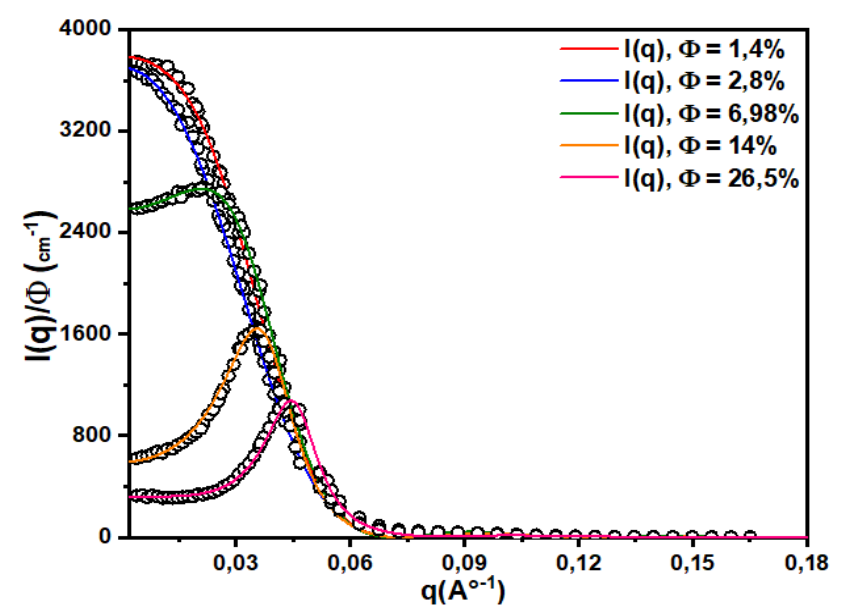

Fig. 5. The SANS spectra of the scattered intensityof bare microemulsion, as a function of $\mathrm{q}$, at different $\Phi(-1.4 \%,-2.8 \%$, $6.98 \%,-14 \%,-26.5 \%)$.

\section{Keywords}

Microemulsion, Small Angle Neutron Scattering (SANS), OrnsteinZernicke (OZ), Hypernetted Chain (HNC), Structure factor S(q).

\section{Received: 06 April 2020}

Revised: 23 April 2020

Accepted: 04 May 2020

\section{References}

1. Hamley, I. W.; Introduction to Soft Matter: Polymers, Colloids, Amphiles and Liquid Crystals, John Wiley and Sons, Ltd, Chichester, 2000, 1-350.

2. Jones, R. A. L.; Soft Condensed Matter, Oxford Master Series in Physics, 2002, 1-208.

3. Raffa, P. Diego Armando, Z. We, Picchioni, F.; Broekhuis, A.A; Polymeric Surfactants: Synthesis, Properties, and Links to Applications, Chemical Reviews, 2015, 115, 16.

4. Bagger-Jörgensen, H.; Coppola, L.; Thuresson, K.; Olsson, U.; Mortensen, K.; Langmuir, 1997, 13, 4204.

5. Bringer, A.; Eisenriegler, E; Schlesener, F; Hanke, A; Eur. Phys. J. $B, 1999,11,101$.

6. Asakura, S.; Oosawa, F.; J. Polym. Sci., 1958, 33, 183.

7. Fleer, G. J.; Cohen Stuart, M. A.; Cosgrove, T.; Scheutjens, J. M.; Vincent, B.; Polymers at interfaces. Am. Chem. Soc., 1996, 118, 297. 


\section{Advanced}

8. de Gennes, P.G.; Advanced in Colloid and Interface Science., 1987, 27, 189.

9. Zilman, A.; Kieffer J.; Molino, F.; Porte, G; Safran, S. A.; Phys. Rev. Lett., 2003, 91, 015901.

10. de Gennes, P. G.; Macromolecules, 1980, 13, 1069.

11. Murat, M.; Grest, G. S.; Phys. Rev. Lett., 1989, 63, 1074.

12. Murat, M.; Grest, G. S.; Macromolecules, 1989, 22, 4054.

13. Malo de Molina, P.; Appavou, M. S.; Gradzielski, M.; Soft Matter, 2014, 10, 5072.

14. Milner, S. T.; Witten, T. A.; Macromolecules, 1992, 25, 5495.

15. Meng, X. X.; Russel, W. B.; Macromolecules, 2003, 36, 10112.

16. Eiser, E.; Klein, J.; Witten, T. A.; Fetters, L. J.; Phys. Rev. Lett, 1999, 82, 5076.

17. Dai L.; Toprakcioglu; C.; Euro. Phys. Lett, 1991, 16, 331.

18. Yethiraj, A.; Hall, C. K.; Macromolecules, 1990, 23, 1865

19. Murat, M.; Great, G. S.; Macromolecules, 1989, 22, 4054.

20. Bhatia, S. R.; Russel, W. B.; Macromolecules, 2000, 33, 5713.

21. Porte, G.; Ligoure, C.; Appell, J.; Aznar, R.; J. Stat. Mech.: Theory Exp., 2006, 05, P05005.

22. Helgeson, M. E.; Wagner, N. J.; J. Chem. Phys, 2011, 135, 084901.

23. Malo de Molina, P.; Herfurth, C.; Laschewsky, A.; Gradzielski, M.; Langmuir, 2012, 28, 15994.

24. Elghazrani, K.; Azougarh, A.; Oberdisse, J.; Filali, M.; Eur. Phys. J. E, 2014, 37, 1

25. Kaczmarski, J. P.; Glass, J. E.; Macromolecules.,1993, 26, 5149.

26. Vorobyova, O.; Yecta, A.; Winnik, M. A.; Lau, W.; Macromolecules, 1998, 31, 8998.

27. Hartmann, P.; Collet, A; Viguier, M.; J. Fluorine. Chem, 1999, 95, 145.

28. Schulman, J. H; Stoeckenius, W.; Prince, L. M.; J. Phys. Chem, 1959, 63, 1677.

29. Hellweg T.; Phase structures of microemulsions, Current Opinion in Colloid and Interface Science, 2002, 7(1-2), 50-56.

30. Bagger-Jorgensen, H.; Olsson, U.; Mortensen K.; Langmuir, 1997, $13,1413$.

31. Safran S. A.; Phys. Rev. A, 1991, 43, 2903

32. Zemb T.; Linder P.; Neutrons, X-ray and Light Scattering Methods Applied to soft Condensed Matter (North Holland Delta Series), First Edition, 2002, Chapt.2, p23.

33. Lairez, D.; J. Phys IV Fr, 1999, 9, 67.

34. Chen, S. H.; Rajagopalan, R.; Micellar Solutions and Microemulsions: Structure, Dynamics, and Statistical Thermodynamics, Springer Science and Business Media, 2012,

35. Borsali, R.; Pecora R.; Soft Matter Characterization, Springer Netherlands, 2008, pp. 723.

36. Appell, J.; Porte, G.; Rawiso, M.; Langmuir, 1998, 14, 4409.

37. Belloni, L.; Colloidal interactions, J. Phys. Condens. Matter, 2000, 12.

38. Belloni, L.; J. Phys. Condens. Matter, 2002, 14, 9323.

39. Russel, W. B.; Saville, D. A., Schowalter, W. R.; Colloidal Dispersions, Cambridge University Press, Cambridge, UK, 1992, 54 (2).

40. Mahanty, J.; Ninham, B.W.; Dispersion Forces, Academic Press, London-New York-San Francisco; 1976, 81(8).

41. Israelachvili, J. N.; Intermolecular and Surfaces Forces, Second Edition: With Applications to Colloidal and Biological Systems (Colloid Science), Academic, 1992, p228.

42. Verwey, E. J. W.; Overbeek, J. TH. G.; Theory of the stability of lyophobic colloids, Dover Books on Chemistry, 1999.

43. Ahfir, R.; Azougarh, A.; Benhamou, M.; Filali, M.; Study of the Colloidal Interactions between Microemulsion Nanodroplets in the Presence of Telechelic Polymers: Theory and Experiment, International Review of Physics, 2014, 8(3).

44. Filali, M.; Aznar, R.; Svenson, M.; Porte, G.; Appell, J.; J. Phys. Chem. B, 1999, 103, 7293.

45. Cabane, B.; Duplessix, R.; J. Phys. France, 1982, 43, 1529.

46. Hansen, J. P; McDonald, I. R.; Theory of Simple Liquids, Second edition, Academic, London, 1986

47. Ornstein, L. S; Zernike, F.; Accidental Deviations of Density and Opalescence at the Critical Point of a Single Substance, Proc. Akad. Sci (Amsterdam), 1914, 17, 793-807.
48. van Leeuwen, J. M. J.; Groenveld, J; De Boer, J.; New method for the calculation of the pair correlation function; I, Physica,1959, 25(7-12), 792-808.

Authors biography

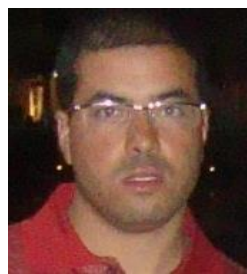

Rachid Ahfir was born in Sefrou/Morocco on 1980. He is a PhD student at the University Sidi Mohammed Ben Abdellah -Fes- Morocco. Her research field of interest is on the colloidal interactions between charged $\mathrm{O} / \mathrm{W}$ microemulsion spherical nanodroplets, in the presence of telechelic polymers PEO-m or PEO- $2 \mathrm{~m}$ by using Small Angle Neutron Scattering with computing studies. He is also interested to the relaxation mode of neutral spherical micelles with dynamic light scattering.

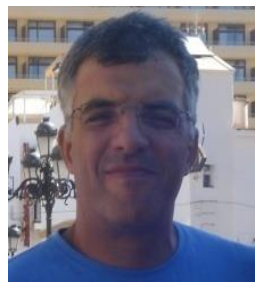

Mohammed Filali was born in Fez/Morocco on 1966. Professor at University Sidi Mohamed Ben Abdellah of Fez (Morocco).

Research Areas: Colloids, Polymer Physics, SoftCondensed Matter. 